Pacific Journal of Mathematics

ESSENTIAL PRODUCTS OF NONSINGULAR RINGS 


\title{
ESSENTIAL PRODUCTS OF NONSINGULAR RINGS
}

\author{
K. R. GOODEARL
}

\begin{abstract}
By an essential product of two rings is meant a subdirect product which contains an essential right ideal of the direct product. The aim of this paper is to investigate the utility of this concept in the study of nonsingular rings. The first section derives some basic properties of essential products and develops some criteria for recognizing essential products. In the second section, a study of the socles of nonsingular modules leads to a theorem that any nonsingular ring is an essential product of a ring with essential socle and a ring with zero socle. The third section is devoted to a theorem which tells when an essential product can be a splitting ring, i.e., a ring such that the singular submodule of any right module is a direct summand. In the final section, this theorem is used to construct two examples of splitting rings of types previously unknown.
\end{abstract}

In this paper all rings are associative with identity, and all modules are unital. We also require that a subring of a ring have the same identity as the ring. Unless otherwise noted, all modules are right modules.

Inasmuch as we use singular and nonsingular modules throughout this paper, we recall the relevant definitions here. Given a ring $R$, we use $\mathscr{S}(R)$ to denote the collection of essential right ideals of $R$; then the singular submodule of a right $R$-module $A$ is the set $Z_{R}(A)=$ $\{a \in A \mid a I=0$ for some $I \in \mathscr{S}(R)\}$. The module $A$ is said to be singular [nonsingular] provided $Z_{R}(A)=A\left[Z_{R}(A)=0\right]$. The singular submodule of $R_{R}$ is a two-sided ideal of $R$, called the right singular ideal of $R$ and denoted $Z_{r}(R) ; R$ is a right nonsingular ring when $Z_{r}(R)=0$.

1. Essential products. Given two right nonsingular rings $R_{1}$ and $R_{2}$, we define an essential product of $R_{1}$ and $R_{2}$ to be any subdirect product $R$ of $R_{1}$ and $R_{2}$ which contains an essential right ideal of $R_{1} \times R_{2}$. [Recall that for $R$ to be a subdirect product of $R_{1}$ and $R_{2}, R$ must be a subring of $R_{1} \times R_{2}$ such that the projections $R \rightarrow R_{1}$ and $R \rightarrow R_{2}$ are both surjective.] The aim of this section is to consider the relationships among singular and nonsingular modules over $R_{1}, R_{2}$, and $R$, and to establish criteria for judging which rings are essential products.

N.B.-For the first three propositions in this section, we assume that $R$ is an essential product of two right nonsingular rings $R_{1}$ and 
$R_{2}$. We define $E_{1} \subset R_{1}$ and $E_{2} \subset R_{2}$ by the conditions $E_{1} \times 0=R \cap$ $\left(R_{1} \times 0\right)$ and $0 \times E_{2}=R \cap\left(0 \times R_{2}\right)$. Inasmuch as $E_{1} \times 0$ is a twosided ideal of $R$ and $R$ is a subdirect product of $R_{1}$ and $R_{2}, E_{1}$ must be a two-sided ideal of $R_{1}$. Likewise, $E_{2}$ is a two-sided ideal of $R_{2}$, hence $E_{1} \times E_{2}$ is a two-sided ideal of $R_{1} \times R_{2}$ as well as a two-sided ideal of $R$. Note that the induced ring homomorphisms $R /\left(E_{1} \times 0\right) \rightarrow R_{2}$ and $R /\left(0 \times E_{2}\right) \rightarrow R_{1}$ are both isomorphisms, from which we conclude that the induced ring homomorphisms

$$
R /\left(E_{1} \times E_{2}\right) \longrightarrow R_{2} / E_{2} \text { and } R /\left(E_{1} \times E_{2}\right) \longrightarrow R_{1} / E_{1}
$$

are also isomorphisms.

Since $R$ is an essential product, it must contain some essential right ideal $I$ of $R_{1} \times R_{2}$. Noting that $I=I_{1} \times I_{2}$ for some $I_{1} \in \mathscr{S}\left(R_{1}\right)$ and $I_{2} \in \mathscr{S}\left(R_{2}\right)$, we infer from $I_{1} \times 0 \leqq E_{1} \times 0$ that $E_{1} \in \mathscr{S}\left(R_{1}\right)$; likewise $E_{2} \in \mathscr{S}\left(R_{2}\right)$. Consequently $E_{1} \times E_{2} \in \mathscr{S}\left(R_{1} \times R_{2}\right)$.

Proposition 1. Let $T=R_{1} \times R_{2}$ and $E=E_{1} \times E_{2}$.

(a) $\mathscr{S}(T)=\left\{K \leqq T_{T} \mid K \cap R \in \mathscr{S}(R)\right\}$.

(b) $\mathscr{S}(R)=\left\{J \leqq R_{R} \mid J E \in \mathscr{S}(T)\right\}$.

(c) $Z_{T}(A)=Z_{R}(A)$ for all $A_{T}$.

(d) $Z_{r}(R)=Z_{R}(T)=0$.

Proof. (a) Suppose that $K \in \mathscr{S}(T)$ and $A \leqq R_{R}$ such that $A \cap$ $(K \cap R)=0$. Then $A E \cap K=0$, hence from $K \in \mathscr{S}(T)$ we obtain $A E=0$. Since $E \in \mathscr{S}(T)$ it follows that $A \leqq Z_{r}(T)=0$, and so $K \cap R \in \mathscr{S}(R)$.

Now let $K \leqq T_{T}$ and assume that $K \cap R \in \mathscr{S}(R)$. If $A \leqq T_{T}$ and $A \cap K=0$, then from $(A \cap R) \cap(K \cap R)=0$ we obtain $A \cap R=0$, hence $A \cap E=0$. Thus $A=0$ and so $K \in \mathscr{S}(T)$.

(b) If $J \leqq R_{R}$ and $J E \in \mathscr{S}(T)$, then $J E \in \mathscr{S}(R)$ by (a), whence $J \in \mathscr{S}(R)$.

Now consider any $J \in \mathscr{S}(R)$. Inasmuch as $E \in \mathscr{S}(T)$ and $Z_{r}(T)=0$, the left annihilator of $E$ in $T$ is zero. In particular, it follows that every nonzero element of $J$ has a nonzero right multiple in $J E$. Thus $J E$ is an essential $R$-submodule of $J$, hence $J E \in \mathscr{S}(R)$, and then $J E \in \mathscr{S}(T)$ by (a).

(c) follows directly from (a) and (b).

(d) According to (c), $Z_{R}(T)=0$, and then $Z_{r}(R)=0$ also.

Letting $Q_{1}$ and $Q_{2}$ denote the respective maximal right quotient rings of $R_{1}$ and $R_{2}$, then $Q_{1} \times Q_{2}$ is the maximal right quotient ring of $R_{1} \times R_{2}$. Inasmuch as $Z_{r}\left(R_{1} \times R_{2}\right)=0$, [4, Theorem $1+2$, p. 69] says that $Q_{1} \times Q_{2}$ is a right self-injective ring.

Proposition 2. $Q_{1} \times Q_{2}$ is also the maximal right quotient ring of $R$. 
Proof. Set $T=R_{1} \times R_{2}, E=E_{1} \times E_{2}$, and $Q=Q_{1} \times Q_{2}$. Note that $T \cap Z_{T}(Q)=Z_{r}(T)=0$, from which we obtain $Z_{T}(Q)=0$.

We first show that $Q$ is a right quotient ring of $R$, i.e., that $Q_{R}$ is a rational extension of $R_{R}$. (See [4, pp. 58,64].) Inasmuch as $Z_{r}(R)=0$, [4, Proposition 5, p. 59] says that it suffices to prove that $Q_{R}$ is an essential extension of $R_{R}$. Thus consider any $A \leqq Q_{R}$ such that $A \cap R=0$. Then $A E \cap E=0$. Since $E$ is an essential right ideal of $T$ it must be an essential $T$-submodule of $Q$, so that we obtain $A E=0$ and $A \leqq Z_{T}(Q)=0$. Therefore $Q$ is a right quotient ring of $R$, hence we many assume that $Q$ is a subring of the maximal right quotient ring $P$ of $R$. The injectivity of $Q_{Q}$ implies that $P_{Q}=$ $Q \oplus B$ for some $B$. Then from $R \cap B=0$ we infer that $B=0$ and $P=Q$.

In view of Proposition 2, we may refer again to [4, Theorem $1+2$, p. 69] and conclude that $\left(Q_{1} \times Q_{2}\right)_{R}$ is an injective hull for $R_{R}$. Now we obtain from [7, Proposition 1, p. 427] the following alternate description of the singular submodule of a right $R$-module $A: Z_{R}(A)=$ $\cap\left\{\operatorname{ker} f \mid f \in \operatorname{Hom}_{R}\left(A, Q_{1} \times Q_{2}\right)\right\}$. In particular, $A$ is singular if and only if $\operatorname{Hom}_{R}\left(A, Q_{1} \times Q_{2}\right)=0$, from which we conclude that any extension of a singular module by a singular module is singular. The corresponding property for nonsingular modules is a consequence of the observation that $A$ is nonsingular if and only if $\operatorname{Hom}_{R}(R / I, A)=0$ for all $I \in \mathscr{S}(R)$.

According to Proposition 1, any nonsingular right $R_{1} \times R_{2}$-module is also a nonsingular right $R$-module. We can view this as saying that the direct sum of a nonsingular right $R_{1}$-module with a nonsingular right $R_{2}$-module gives a nonsingular right $R$-module. The converse is false unless $R$ is actually the direct product of $R_{1}$ and $R_{2}$, but according to the next proposition we can at least realize any nonsingular right $R$-module as an extension of a nonsingular right $R_{1}$-module by a nonsingular right $R_{2}$-module.

Proposition 3. (a) $A$ right $R$-module $A$ is nonsingular if and only if it has a submodule $A^{\prime}$ such that $A^{\prime}$ is a nonsingular right $R_{1}$-module and $A / A^{\prime}$ is a nonsingular right $R_{L^{-}}$module.

(b) A right $R$-module $C$ is singular if and only if it has a submodule $C^{\prime}$ such that $C^{\prime}$ is a singular right $R_{1}$-module and $C / C^{\prime}$ is a singular right $R_{2}$-module.

Proof. (a) If $A$ has such a submodule $A^{\prime}$, then according to Proposition $1, A^{\prime}$ and $A / A^{\prime}$ are both nonsingular $R$-modules, hence $A$ must be nonsingular. 
Now assume that $A$ is nonsingular. In view of the discussion above, the intersection of the kernels of the homomorphisms from $A$ into $Q_{1} \times Q_{2}$ must be zero. Thus we may assume that $A$ is a submodule of some direct product $B$ of copies of $Q_{1} \times Q_{2}$. Note that $B=B_{1} \oplus B_{2}$, where $B_{i}$ is a direct product of copies of $Q_{i}$; since $Q_{i}$ is the maximal right quotient ring of $R_{i}, B_{i}$ is a nonsingular right $R_{i}$-module. Consequently, $A^{\prime}=A \cap B_{1}$ is a nonsingular right $R_{1^{-}}$ module, and $A / A^{\prime}$ is a nonsingular right $R_{2}$-module.

(b) If $C$ has such a submodule $C^{\prime}$, then according to Proposition 1, $C^{\prime}$ and $C / C^{\prime}$ are both singular $R$-modules, whence $C$ must be singular.

Conversely, assume that $C$ is singular. Clearly $C^{\prime}=C\left(E_{1} \times 0\right)$ is a right $R_{1}$-module [because $C^{\prime}\left(0 \times E_{2}\right)=0$ ] and $C / C^{\prime}$ is a right $R_{2}$-module. Inasmuch as $C^{\prime}$ and $C / C^{\prime}$ are singular $R$-modules, Proposition 1 says that $C^{\prime}$ is a singular $R_{1}$-module and $C / C^{\prime}$ is a singular $R_{2}$-module.

N. B.-We now drop the a priori assumption that $R$ is an essential product of $R_{1}$ and $R_{2}$, in order to find conditions under which $R$ can be such an essential product.

THEOREM 4. Let $R_{1}$ and $R_{2}$ be right nonsingular rings, $R$ any subring of $R_{1} \times R_{2}$. Then the following conditions are equivalent:

(a) $R$ is an essential product of $R_{1}$ and $R_{2}$.

(b) There exist two-sided ideals $E_{1} \in \mathscr{S}\left(R_{1}\right)$ and $E_{2} \in \mathscr{S}\left(R_{2}\right)$, and a ring isomorphism $\phi: R_{1} / E_{1} \rightarrow R_{2} / E_{2}$, such that

$$
R=\left\{(x, y) \in R_{1} \times R_{2} \mid \phi\left(x+E_{1}\right)=y+E_{2}\right\} .
$$

(c) $R$ is a subdirect product of $R_{1}$ and $R_{2}, R /\left[R \cap\left(R_{1} \times 0\right)\right]$ and $R /\left[R \cap\left(0 \times R_{2}\right)\right]$ are both nonsingular right $R$-modules, and

$$
\left[R \cap\left(R_{1} \times 0\right)\right]+\left[R \cap\left(0 \times R_{2}\right)\right] \in \mathscr{S}(R) .
$$

Proof. Set $H_{1}=R \cap\left(R_{1} \times 0\right)$ and $H_{2}=R \cap\left(0 \times R_{2}\right)$, both of which are two-sided ideals of $R$.

(a) $\Rightarrow$ (c): By definition, $R$ is a subdirect product of $R_{1}$ and $R_{2}$. Set $E_{1} \times 0=H_{1}$ and $0 \times E_{2}=H_{2}$ : then $H_{1}+H_{2}=E_{1} \times E_{2}$, which in the discussion prior to Proposition 1 is shown to belong to $\mathscr{S}\left(R_{1} \times R_{2}\right)$. Quoting part (a) of Proposition 1, we see that $H_{1}+H_{2} \in \mathscr{S}(R)$. As for the modules $\left(R / H_{1}\right)_{R}$ and $\left(R / H_{2}\right)_{R}$, they are both isomorphic to submodules of $\left(R_{1} \times R_{2}\right)_{R}$, which is nonsingular by Proposition 1 .

(c) $\Rightarrow$ (b): Since $R$ is a subdirect product of $R_{1}$ and $R_{2}$, it follows as in the discussion before Proposition 1 that there exist two-sided ideals $E_{1}$ in $R_{1}$ and $E_{2}$ in $R_{2}$ such that $E_{1} \times 0=H_{1}$ and $0 \times E_{2}=H_{2}$, 
and that the induced maps $f: R /\left(E_{1} \times E_{2}\right) \rightarrow R_{1} / E_{1}$ and $g: R /\left(E_{1} \times E_{2}\right) \rightarrow$ $R_{2} / E_{2}$ are ring isomorphisms. Setting $\phi=g f^{-1}$, we check that

$$
R=\left\{(x, y) \in R_{1} \times R_{2} \mid \dot{\phi}\left(x+E_{1}\right)=y+E_{2}\right\} .
$$

Inasmuch as $\left(R / H_{1}\right)_{R}$ is nonsingular and $H_{1}+H_{2} \in \mathscr{S}(R)$, no nonzero element of $R / H_{1}$ is annihilated on the right by $H_{1}+H_{2}$. Noting that the isomorphism $R / H_{1} \rightarrow R_{2}$ [induced by the projection $R \rightarrow R_{2}$ ] carries $\left(H_{1}+H_{2}\right) / H_{1}$ onto $E_{2}$, we infer that the left annihilator of $E_{2}$ in $R_{2}$ is zero. Since $E_{2}$ is a two-sided ideal of $R_{2}$, it follows that every nonzero element of $R_{2}$ has a nonzero right multiple in $E_{2}$, whence $E_{2} \in \mathscr{S}\left(R_{2}\right)$. Likewise, $E_{1} \in \mathscr{S}\left(R_{1}\right)$.

(b) $\Rightarrow$ (a): It is easy to check that under these hypotheses, $R$ is a subdirect product of $R_{1}$ and $R_{2}$. Also, $R$ contains $E_{1} \times E_{2}$, which is an essential right ideal of $R_{1} \times R_{2}$, hence $R$ is an essential product.

Theorem 4 may be thought of as characterizing "external" essential products. As an immediate consequence of the equivalence of (a) and (c), we also get the following characterization of "internal" essential products.

COROLlaRY 5. Let $R_{1}$ and $R_{2}$ be right nonsingular rings. Then a ring $R$ is isomorphic to an essential product of $R_{1}$ and $R_{2}$ if and only if there exist two-sided ideals $H_{1}$ and $H_{2}$ in $R$ such that

(a) $H_{1} \cap H_{2}=0$.

(b) $R / H_{1} \cong R_{2}$ and $R / H_{2} \cong R_{1}$.

(c) $\left(R / H_{1}\right)_{R}$ and $\left(R / H_{2}\right)_{R}$ are both nonsingular.

(d) $H_{1}+H_{2} \in \mathscr{S}(R)$.

2. Socles and antisocles. The purpose of this section is to prove that any right nonsingular ring is isomorphic to an essential product of a ring with essential socle and a ring with zero socle. To this end we first develop some results about socles of nonsingular modules over an arbitrary ring $R$.

For any simple right $R$-module $R / M$, there are only two choices for the submodule $Z_{l i}(R / M)$, hence $R / M$ must be either singular or nonsingular. In case it is nonsingular, then $M \notin \mathscr{S}(R)$, whence $M \cap$ $I=0$ for some nonzero right ideal $I$ of $R$. By the maximality of $M, R=M \oplus I$. Thus we see that every simple right $R$-module is either singular or projective. Consequently, every nonsingular semisimple right $R$-module is projective.

Proposition 6. Let $J=\operatorname{soc}\left(R_{R}\right)$. Then $\operatorname{soc}(A)=A J$ for any nonsingular right $R$-module $A$.

Proof. The module $A J$ is clearly semisimple, hence $A J \leqq \operatorname{soc}(A)$. 
On the other hand, any simple submodule $B$ of $A$ is projective and thus is isomorphic to $e R$ for some idempotent $e \in R$, whence $B=$ $B e R \leqq A J$.

Proposition 7. Let $A$ be any nonsingular right $R$-module, $J=$ $\operatorname{soc}\left(R_{R}\right)$. socle.

(a) $B=\{a \in A \mid a J=0\}$ is the largest submodule of $A$ with zero

(b) $A / B$ has essential socle.

(c) $A / B$ is nonsingular.

Proof. (a) is immediate from Proposition 6.

(b) If $N / B$ is any nonzero submodule of $A / B$, then since $N \not B$ we must have $N J \neq 0$. We infer from the semisimplicity of $N J$ that $N J \cap B=0$, whence $(N J+B) / B \neq 0$ and thus $[N / B] \cap[(A J+B) / B] \neq 0$. Therefore $(A J+B) / B$ is an essential submodule of $A / B$. Inasmuch as $(A J+B) / B$ is semisimple, we conclude that $A / B$ has essential socle.

(c) Noting that $A J \cap B=0$, we see that $(A J+B) / B \cong A J$, which is a nonsingular module. Thus $A / B$ is an essential extension of a nonsingular module, hence $A / B$ must be nonsingular.

According to Proposition 7, a nonsingular right $R$-module $A$ always has a largest submodule with zero socle; Professor Kaplansky has suggested the name antisocle for this submodule of $A$. We now proceed in a similar manner to show that $A$ also has a largest submodule with essential socle. We refrain from introducing a name for this submodule, since in the presence of a suitable notion of closure it is describable simply as the closure of soc $(A)$.

Proposition 8. Let $A$ be any nonsingular right $R$-module. Set $J=\operatorname{soc}\left(R_{R}\right)$, and let $H$ be the left annihilator of $J$ in $R$.

(a) $C=\{a \in A \mid a H=0\}$ is the largest submodule of $A$ with essential socle.

(b) $A / C$ is nonsingular.

(c) $A / C$ has zero socle.

Proof. Inasmuch as $J$ is a two-sided ideal of $R, H$ is a two-sided ideal also. Note that $J+H \in \mathscr{S}(R)$ : For if $x \in R \backslash H$, then $x J \neq 0$ and thus $x$ has a nonzero right multiple in $J+H$.

(a) will follow immediately from the following claim: A nonsingular right $R$-module $B$ has essential socle if and only if $B H=0$.

First suppose that $B$ has essential socle, i.e., that $B J$ is essential in $B$. Inasmuch as $B H \cap B J$ is semisimple, we obtain $B H \cap B J=$ $(B H \cap B J) J \leqq B H J=0$, from which $B H=0$ follows. Conversely, if 
$B H=0$, then since $J+H \in \mathscr{S}(R)$ we must have $x J \neq 0$ for all nonzero $x \in B$, from which we infer that $B J$ is essential in $B$.

(b) Inasmuch as $A H J=0$, we see that $A H$ has zero socle, whence $A H \cap \operatorname{soc}(C)=0$ and thus $A H \cap C=0$. In particular, we find that $(A H+C) / C \cong A H$, which is a nonsingular module.

If $N / C$ is any nonzero submodule of $A / C$, then since $N \$ C$ we must have $N H \neq 0$. Since $N H \cap C \leqq A H \cap C=0$, it follows that $(N H+C) / C \neq 0$, whence $[N / C] \cap[(A H+C) / C] \neq 0$. Thus $A / C$ is an essential extension of the nonsingular module $(A H+C) / C$, so $A / C$ must be nonsingular.

(c) According to (a), $\operatorname{soc}(A) \leqq C$. Inasmuch as the nonsingularity of $A / C$ implies that soc $(A / C)$ is projective, we conclude that $\operatorname{soc}(A / C)=0$.

When $R$ is presented as an essential product of rings $R_{1}$ and $R_{2}$, both $R_{1}$ and $R_{2}$ are factor rings of $R$. Thus we need to know that certain factor rings of $R$ are nonsingular, for which reason we introduce the next proposition. The proof is routine, and may be found in [8, Proposition 1.11].

Proposition 9. Let $H$ be a two-sided ideal of $R$ such that $(R / H)_{R}$ is nonsingular.

(a) $\mathscr{S}(R / H)=\{I / H \mid H \leqq I$ and $I \in \mathscr{S}(R)\}$.

(b) $Z_{R / H}(A)=Z_{R}(A)$ for all $A_{R / I I}$.

(c) $Z_{r}(R / H)=0$.

TheOREM 10. Let $R$ be any right nonsingular ring. Then $R$ is isomorphic to an essential product of two right nonsingular rings $R_{1}$ and $R_{2}$ such that $R_{1}$ has essential right socle and $R_{2}$ has zero right socle.

Proof. Let $J=\operatorname{soc}\left(R_{R}\right), H$ the left annihilator of $J$ in $R, K$ the left annihilator of $H$ in $R$. Since $J$ is a two-sided ideal, so are $H$ and $K$. The modules $(R / H)_{R}$ and $(R / K)_{R}$ are nonsingular according to Propositions 7 and 8 , hence by Proposition $9, R_{1}=R / H$ and $R_{2}=$ $R / K$ are both right nonsingular rings.

Referring to Propositions 7 and 8 again, we see that $H_{R}$ has zero socle while $K_{R}$ has essential socle, hence $H \cap K=0$. We must also note that $H+K \in \mathscr{S}(R)$ : For if $X \in R \backslash K$, then $x H \neq 0$ and so $x$ has a nonzero right multiple in $H+K$. According to Corollary 5, we obtain that $R$ is isomorphic to an essential product of $R_{1}$ and $R_{2}$.

Inasmuch as $(R / H)_{R}$ has essential socle by Proposition $7,(R / H)_{R / H}$ must have essential socle. Similarly, $(R / K)_{R}$ has zero socle by Proposition 8 , whence $(R / K)_{R / K}$ has zero socle.

3. Splitting rings. This section is concerned with the question 
of when an essential product can be a splitting ring, by which we mean a ring $R$ such that for every right $R$-module $A, Z_{R}(A)$ is a direct summand of $A$. As is noted in [2, Proposition 1.12], $R$ is a splitting ring if and only if $\operatorname{Ext}_{R}^{1}(A, C)=0$ for all nonsingular $A_{R}$ and all singular $C_{R}$.

N.B.-Throughout this section, we assume that $R$ is an essential product of two right nonsingular rings $R_{1}$ and $R_{2}$. Setting $E_{1} \times 0=$ $R \cap\left(R_{1} \times 0\right)$ and $0 \times E_{2}=R \cap\left(0 \times R_{2}\right)$, we recall from $\S 1$ that each $E_{i}$ is a two-sided ideal in $\mathscr{S}\left(R_{i}\right)$. According to Proposition 1 , we also have $E_{1} \times E_{2} \in \mathscr{S}(R)$. Setting $H_{1}=E_{1} \times 0$ and $H_{2}=0 \times E_{2}$, we recall that $R / H_{1} \cong R_{2}$ and $R / H_{2} \cong R_{1}$, while from Theorem 4 we obtain that $R / H_{1}$ and $R / H_{2}$ are nonsingular right $R$-modules.

Lemma 11. Let $T$ be a splitting ring, $H$ a two-sided ideal in $\mathscr{S}(T), A$ any nonsingular right $T$-module.

(a) $\operatorname{Tor}_{1}^{T}(A, T / H)=0$.

(b) $A / A H$ is a projective right $(T / H)$-module.

Proof. (a) Choosing a divisible abelian group $D$ which contains $\operatorname{Tor}_{1}^{T}(A, T / H)$, we note that $\operatorname{Hom}_{Z}(T / H, D)$ is a two-sided $T$-module. As a right $T$-module, $\operatorname{Hom}_{Z}(T / H, D)$ is singular because $H \in \mathscr{S}(T)$, whence $\operatorname{Ext}_{T}^{1}\left(A, \operatorname{Hom}_{Z}(T / H, D)\right)=0$. According to [1, Proposition 5.1, p. 120], we obtain $\operatorname{Hom}_{Z}\left(\operatorname{Tor}_{1}^{T}(A, T / H), D\right)=0$, and thus

$$
\operatorname{Tor}_{1}^{T}(A, T / H)=0 \text {. }
$$

(b) Choose an exact sequence $S: 0 \rightarrow K \rightarrow F \rightarrow A / A H \rightarrow 0$, where $F$ is a free right $(T / H)$-module. Inasmuch as $K H=0$ and $H \in \mathscr{S}(T)$, $K$ is a singular right $T$-module. Thus $\operatorname{Ext}_{T}^{1}(A, K)=0$, hence the natural map $p: A \rightarrow A / A H$ lifts to a map $f: A \rightarrow F$. Since $F H=0, f$ induces a map $g: A / A H \rightarrow F$ which splits $S$.

THEOREM 12. $R$ is a splitting ring if and only if

(a) $R_{1}$ and $R_{2}$ are splitting rings.

(b) If $L_{i}$ is any essential right $R_{i}$-submodule of $E_{i}$, then $E_{i} / L_{i}$ is a direct summand of $R_{i} / L_{i}$.

Proof. Assume that $R$ is a splitting ring. If $H=H_{1}$ or $H=H_{2}$, and $A$ is any right $(R / H)$-module, then $Z_{R / H}(A)=Z_{R}(A)$ by Proposition 9 , hence $Z_{R / H}(A)$ is a summand of $A$. Therefore $R / H_{1}$ and $R / H_{2}$ are splitting rings; i.e., $R_{2}$ and $R_{1}$ are splitting rings.

Next consider any essential right $R_{1}$-submodule $L_{1} \leqq E_{1}$. Noting that $L_{1} \times 0$ is an essential right $R$-submodule of $H_{1}$, we see that $H_{1} /\left(L_{1} \times 0\right)$ is a singular right $R$-module. Inasmuch as $\left(R / H_{1}\right)_{R}$ is 
nonsingular, the exact sequence

$$
0 \longrightarrow H_{1} /\left(L_{1} \times 0\right) \longrightarrow R /\left(L_{1} \times 0\right) \longrightarrow R / H_{1} \longrightarrow 0
$$

must split. Tensoring with $R / H_{2}$, we obtain another split exact sequence

$$
0 \longrightarrow\left(E_{1} \times 0\right) /\left(L_{1} \times 0\right) \longrightarrow R /\left(L_{1} \times E_{2}\right) \longrightarrow R /\left(E_{1} \times E_{2}\right) \longrightarrow 0,
$$

from which we infer that $E_{1} / L_{1}$ is a summand of $R_{1} / L_{1}$. By symmetry, $E_{2} / L_{2}$ is also a summand of $R_{2} / L_{2}$ whenever $L_{2}$ is an essential right $R_{2}$-submodule of $E_{2}$.

Now suppose that (a) and (b) hold. Inasmuch as $E_{1} \in \mathscr{S}\left(R_{1}\right)$ and $Z_{r}\left(R_{1}\right)=0$, the left annihilator of $E_{1}$ in $R_{1}$ is zero. In particular, every nonzero element of $E_{1}$ has a nonzero right multiple in $E_{1}^{2}$, hence $E_{1}^{2}$ is an essential right $R_{1}$-submodule of $E_{1}$. According to (b), $E_{1} / E_{1}^{2}$ must be a summand of $R_{1} / E_{1}^{2}$, from which we infer that $E_{1}=E_{1}^{2}$. Thus $H_{1}=H_{1}^{2}$, and likewise $H_{2}=H_{2}^{2}$.

We must show that $\operatorname{Ext}_{R}^{1}(A, C)=0$ for any nonsingular $A_{R}$ and any singular $C_{R}$. By Proposition $3, C$ has a submodule $C^{\prime}$ such that $C^{\prime}$ is a singular right $R_{1}$-module and $C / C^{\prime}$ is a singular right $R_{2^{-}}$ module. Since it suffices to prove that

$$
\operatorname{Ext}_{R}^{1}\left(A, C^{\prime}\right)=0 \text { and } \operatorname{Ext}_{R}^{1}\left(A, C / C^{\prime}\right)=0,
$$

we may thus assume that $C$ is a singular module over either $R_{1}$ or $R_{2}$. In view of symmetry, we need only consider the case when $C$ is a singular right $R_{1}$-module.

Proposition 3 also says that $A$ has a submodule $A^{\prime}$ such that $A^{\prime}$ is a nonsingular right $R_{1}$-module and $A / A^{\prime}$ is a nonsingular right $R_{2}$ module. Inasmuch as it suffices to show that $\operatorname{Ext}_{R}^{1}\left(A^{\prime}, C\right)=0$ and $\operatorname{Ext}_{R}^{1}\left(A / A^{\prime}, C\right)=0$, we may thus assume that $A$ is a nonsingular module over either $R_{1}$ or $R_{2}$.

Case $I . A$ is a nonsingular right $R_{1}$-module.

Consider any exact sequence $S: 0 \rightarrow C \rightarrow B \rightarrow A \rightarrow 0$ of right $R$ modules. Since $C$ and $A$ are both $R_{1}$-modules, we infer from the relation $H_{2}=H_{2}^{2}$ that $B H_{2}=0$ also; i.e., that $S$ is an exact sequence of $R_{1}$-modules. Thus $S$ must split, because $R_{1}$ is a splitting ring.

Case II. $A$ is a nonsingular right $R_{2}$-module.

Inasmuch as $R_{2}$ is a splitting ring and $E_{2} \in \mathscr{S}\left(R_{2}\right)$, Lemma 11 implies that $\operatorname{Tor}_{1}^{R_{2}}\left(A, R_{2} / E_{2}\right)=0$. Equivalently,

$$
\operatorname{Tor}_{1}^{R / H_{1}}\left(A, R /\left(H_{1} \oplus H_{2}\right)\right)=0 \text {, }
$$

from which we infer that $\operatorname{Tor}_{1}^{R}\left(A, R /\left(H_{1} \oplus H_{2}\right)\right)=0$. It follows that 
the map $A \bigotimes_{R} H_{2} \rightarrow A \bigotimes_{R}\left(H_{1} \oplus H_{2}\right) \rightarrow A$ is a monomorphism, hence $\operatorname{Tor}_{1}^{R}\left(A, R / H_{2}\right)=0$.

Consider any exact sequence $S: 0 \rightarrow C \rightarrow B \rightarrow A \rightarrow 0$ of right $R$ modules. Inasmuch as $C H_{2}=0$ and $\operatorname{Tor}_{1}^{R}\left(A, R / H_{2}\right)=0$, we obtain another exact sequence $S^{*}: 0 \rightarrow C \rightarrow B / B H_{2} \rightarrow A / A H_{2} \rightarrow 0$. An easy diagram chase shows that $S$ will split if $S^{*}$ does, so it suffices to prove that $\operatorname{Ext}_{R}^{1}\left(A / A H_{2}, C\right)=0$. According to Lemma 11, $A / A E_{2}$ is a projective right $\left(R_{2} / E_{2}\right)$-module. Noting that $A H_{1}=0$, it follows that $A / A H_{2}$ is a projective right module over $R /\left(H_{1}+H_{2}\right)$. Therefore to get $\operatorname{Ext}_{R}^{1}\left(A / A H_{2}, C\right)=0$, it suffices to prove that

$$
\operatorname{Ext}_{R}^{1}\left(R /\left(H_{1}+H_{2}\right), C\right)=0 .
$$

Thus consider any map $f: H_{1}+H_{2} \rightarrow C$. Noting that $C_{2}=0$ and $H_{2}=H_{2}^{2}$, we see that $H_{2} \leqq \operatorname{ker} f$. Inasmuch as $f$ induces an isomorphism of $\left[\left(H_{1}+H_{2}\right) / H_{2}\right] /\left[(\operatorname{ker} f) / H_{2}\right]$ onto a submodule of $C$, we see that $\left[\left(H_{1}+H_{2}\right) / H_{2}\right] /\left[(\operatorname{ker} f) / H_{2}\right]$ must be a singular right $\left(R / H_{2}\right)$-module. Since also $Z_{r}\left(R / H_{2}\right)=0$, it follows easily that $(\operatorname{ker} f) / H_{2}$ is an essential submodule of $\left(H_{1}+H_{2}\right) / H_{2}$. Considering that the isomorphism of $R / H_{2}$ onto $R_{1}$ carries $\left(H_{1}+H_{2}\right) / H_{2}$ onto $E_{1}$, we obtain from (b) that

$$
\left[\left(H_{1}+H_{2}\right) / H_{2}\right] /\left[(\operatorname{ker} f) / H_{2}\right]
$$

must be a direct summand of $\left[R / H_{2}\right] /\left[(\operatorname{ker} f) / H_{2}\right]$. Thus $\left(H_{1}+H_{2}\right) /$ (ker $f$ ) is a summand of $R /(\operatorname{ker} f$ ), hence $f$ extends to a map $R \rightarrow C$. Therefore $\operatorname{Ext}_{R}^{1}\left(R /\left(H_{1}+H_{2}\right), C\right)=0$.

The combination of Theorems 12 and 10 reduces the problem of characterizing splitting rings to characterizing those with either zero socle or essential socle. Since the splitting rings with essential socle have already been characterized [8, Corollary 5.4], only the case of zero socle remains.

4. ExAmples. In this section we indicate the usefulness of Theorem 12 in constructing splitting rings. The first example shows that essential products [as opposed to direct products] are definitely needed in the study of splitting rings, for this example is a splitting ring which cannot be decomposed into a direct product of a ring with essential socle and a ring with zero socle. The second example shows that a splitting ring with zero socle can have global dimension 2 .

For both examples, we start with a left and right principal ideal domain $C$ such that $C$ is a simple ring but not a division ring, and such that every simple right $C$-module is injective. (Examples of such rings are constructed in [3].) In fact, all singular right $C$ modules are injective, which is proved in [8] in the discussion following Theorem 3.9. Thus $C$ is certainly a splitting ring; however, it 
cannot be a factor in a nontrivial essential product because it has no nontrivial two-sided ideals. For this reason, we choose some maximal right ideal $M$ of $C$ and turn to the idealizer of $M$ in $C$, that is, to the ring $I=\{c \in C \mid c M \leqq M\}$. In the following lemma, we establish the properties of $I$ and $M$ which are needed for our constructions.

LEMma 13. (a) $I$ is a right nonsingular right Noetherian ring with zero right socle.

(b) I is a splitting ring.

(c) $M$ is a two-sided ideal in $\mathscr{S}(I)$, and $I / M$ is a division ring.

(d) If $L$ is any essential submodule of $M_{I}$, then $M / L$ is a direct summand of $I / L$.

Proof. (a) It is pointed out in [6] that $I$ is a right (and left) Noetherian integral domain in which $M$ is a nontrivial two-sided ideal. Inasmuch as $I$ is a domain it must be nonsingular; since it is not a division ring, we must also have $\operatorname{soc}\left(I_{I}\right)=0$.

(b) This is [6, Lemma 2]. (See also [9].)

(c) We observe from the definition of $I$ that $I / M$ is isomorphic to the endomorphism ring of the simple module $(C / M)_{C}$.

(d) Inasmuch as $L M$ is a nonzero right ideal of the right Ore domain $C$, we infer that $L M \in \mathscr{S}(C)$, and hence that $M / L M$ is a singular right $C$-module. We have already noted that all singular right $C$-modules are injective; thus $M / L M$ must be a direct summand of $C / L M$. Two applications of the modular law now show that, first, $M / L M$ is a summand of $I / L M$, and second, that $M / L$ is a summand of $I / L$.

EXAMPLE 1. Let $R$ be the "matrix ring" $\left(\begin{array}{ll}I / M & 0 \\ I / M & I\end{array}\right)$. Then

(a) $R$ is a right nonsingular splitting ring.

(b) $\operatorname{soc}\left(R_{R}\right)$ is neither zero nor essential in $R$.

(c) $R$ is indecomposable as a ring.

(d) $R$ cannot be the direct product of a ring with zero right socle and a ring with essential right socle.

Proof. (a) Set $R_{1}=\left(\begin{array}{cc}I / M & 0 \\ I / M & I / M\end{array}\right), E_{1}=\left(\begin{array}{cc}I / M & 0 \\ I / M & 0\end{array}\right), R_{2}=I, E_{2}=M$. Each $E_{i}$ is a two-sided ideal of $R_{i}$, and we may identify $R_{1} / E_{1}$ with $R_{2} / E_{2}$ in the obvious manner. Now $R$ is isomorphic to the ring $R^{\prime}=$ $\left\{(x, y) \in R_{1} \times R_{2} \mid x+E_{1}=y+E_{2}\right\}$, hence it suffices to show that $R^{\prime}$ is a right nonsingular splitting ring. We know from Lemma 13 that $Z_{r}\left(R_{2}\right)=0$ and $E_{2} \in \mathscr{S}\left(R_{2}\right)$, and it is easy to check that $E_{1} \in \mathscr{S}\left(R_{1}\right)$. Inasmuch as $I / M$ is a division ring, it follows easily that every ele- 
ment of $\mathscr{S}\left(R_{1}\right)$ contains $E_{1}$, from which we infer that $Z_{r}\left(R_{1}\right)=0$. Now according to Theorem $4, R^{\prime}$ is an essential product of $R_{1}$ and $R_{2}$. By Proposition $1, Z_{r}\left(R^{\prime}\right)=0$.

Since $I / M$ is a division ring, [8, Theorem 2.15] says that all nonsingular right $R_{1}$-modules are projective, whence $R_{1}$ must be a splitting ring. [Alternatively, it can be shown that all singular right $R_{1}$-modules are injective. (See [8, Chapter 3].)] By Lemma 13, $R_{2}$ is a splitting ring too.

If $L_{1}$ is any essential right $R_{1}$-submodule of $E_{1}$, it is easy to check that $L_{1}=E_{1}$, hence $E_{1} / L_{1}$ is certainly a summand of $R_{1} / L_{1}$. The corresponding property in $R_{2}$ is proved in Lemma 13, whence from Theorem 12 we see that $R^{\prime}$ is a splitting ring.

(b) Since $\left(\begin{array}{cc}I / M & 0 \\ 0 & 0\end{array}\right)$ is a simple right ideal of $R, \operatorname{soc}\left(R_{R}\right) \neq 0$. By Lemma 13, we have soc $\left(I_{l}\right)=0$, from which we infer that $\left(\begin{array}{ll}0 & 0 \\ 0 & M\end{array}\right)$ is a nonzero right ideal of $R$ which has no simple submodules. Thus $\operatorname{soc}\left(R_{R}\right)$ is not essential in $R_{R}$.

(c) Noting that $I$ is a domain and $I / M$ is a division ring, we see that neither $I$ nor $I / M$ has any nontrivial idempotents. It follows easily that the only nontrivial idempotents in $R$ are of the form $\left(\begin{array}{ll}1 & 0 \\ x & 0\end{array}\right)$ or $\left(\begin{array}{ll}0 & 0 \\ x & 1\end{array}\right)$, neither of which is central in $R$. Thus $R$ cannot be the direct product of two nonzero rings.

$(d)$ is immediate from (b) and (c).

According to [2, Theorem 2.1], a commutative splitting ring has global dimension at most one, while [10, Theorem 2.2] shows that the right global dimension of a noncommutative splitting ring is at most 2. Examples have been constructed of splitting rings with right global dimension 2 ([6] and [8, Example 5.11]), but both of these examples have nonzero socle. Our next example shows that even with zero socle, a splitting ring can have right global dimension 2 .

This example also disproves a conjecture in [5] concerning the finitely generated splitting property (FGSP). [A ring has FGSP provided the singular submodule of any finitely generated right module is a direct summand.] In the case of a right nonsingular ring with zero right socle, this conjecture reduces to the assertion that such a ring has FGSP if and only if all finitely generated nonsingular right modules are projective. Our example has FGSP because it is a splitting ring, but it has a finitely generated nonsingular right module which is not even flat.

Example 2. Let $R=\{(x, y) \in I \times I \mid x+M=y+M\}$.

(a) $R$ is a right nonsingular splitting ring.

(b) $\operatorname{soc}\left(R_{R}\right)=0$.

(c ) r.gl.dim. $(R)=G W D(R)=2$. 
(d) Not all finitely generated nonsingular right $R$-modules are flat.

Proof. (a) Inasmuch as $Z_{r}(I)=0$ and $M$ is a two-sided ideal in $\mathscr{S}(I)$, Theorem 4 shows that $R$ is an essential product of $I$ with itself. By Proposition $1, Z_{r}(R)=0$. Using Lemma 13 with Theorem 12, we see that $R$ is a splitting ring.

(b) Observing that any simple submodule of $(M \times M)_{R}$ would have to be contained in $\left[\operatorname{soc}\left(I_{I}\right)\right] \times\left[\operatorname{soc}\left(I_{I}\right)\right]$, which is zero by Lemma 13, we obtain $(M \times M) \cap \operatorname{soc}\left(R_{R}\right)=0$. Recalling from Proposition 1 that $M \times M \in \mathscr{S}(R)$, we see that $\operatorname{soc}\left(R_{R}\right)=0$.

(c) Since $R$ is a splitting ring, [10, Theorem 2.2] says that r.gl.dim. $(R) \leqq 2$. Inasmuch as $G W D(R) \leqq$ r.gl.dim. $(R)$, it thus suffices to show that $G W D(R)>1$.

Choose a nonzero $m \in M$ and set $A=(m, 0) R$. Noting that $m$ is a non-zero-divisor in $I$, we see that the right annihilator of $(m, 0)$ in $R$ is $0 \times M$, whence $A \cong R /(0 \times M)$. According to Lemma 13, $M_{I}$ is finitely generated, hence $(0 \times M)_{R}$ is finitely generated and $A_{R}$ is finitely presented. Observing that $0 \times M$ contains no nonzero idempotents, we see that $A$ is not projective. Inasmuch as $A$ is finitely presented, we conclude that $A$ is a right ideal of $R$ which is not flat, whence $G W D(R)>1$.

(d) Since all right ideals of $R$ are nonsingular, this is immediate from (c).

\section{REFERENCES}

1. H. Cartan and S. Eilenberg, Homological Algebra, Princeton University Press (1956).

2. V.C. Cateforis and F. L. Sandomierski, The singular submodule splits off, J. Algebra, 10 (1968), 149-165.

3. J. H. Cozzens, Homological properties of the ring of differential polynomials, Bull. Amer. Math. Soc., 76 (1970), 75-79.

4. C. Faith, Lectures on Injective Modules and Quotient Rings, Springer-Verlag Lecture Notes No. 49.

5. J. D. Fuelberth and M. L. Teply, The singular submodule of a finitely generated module splits off, Pacific J. Math., 40 (1972), 73-82.

6. J. D. Fuelberth and M. L. Teply, A splitting ring of global dimension two, Proc. Amer. Math. Soc., 35 (1972), 317-324.

7. E. R. Gentile, Singular submodule and injective hull, Indag. Math., 24 (1962), 426-433.

8. K. R. Goodearl, Singular torsion and the splitting properties, Amer. Math. Soc. Memoirs No. 124.

9. - Idealizers and nonsingular rings, (to appear).

10. M. L. Teply, Homological dimension and splitting torsion theories, Pacific J. Math..

34 (1970), 193-205.

Received February 25, 1972.

UNIVERSity of CHICAGO

Current address: University of Utah. 



\section{PACIFIC JOURNAL OF MATHEMATICS}

\section{EDITORS}

\author{
H. SAMELson \\ Stanford University \\ Stanford, California 94305 \\ C. R. HоввY \\ University of Washington \\ Seattle, Washington 98105
}

J. DugundJI

Department of Mathematics

University of Southern California

Los Angeles, California 90007

RICHARD ARENS

University of California

Los Angeles, California 90024

\section{ASSOCIATE EDITORS}
E. F. BeCKENBACH
B. H. NEUMANN
F. WOLF
K. YoshidA

\section{SUPPORTING INSTITUTIONS}

UNIVERSITY OF BRITISH COLUMBIA

CALIFORNIA INSTITUTE OF TECHNOLOGY

UNIVERSITY OF CALIFORNIA

MONTANA STATE UNIVERSITY

UNIVERSITY OF NEVADA

NEW MEXICO STATE UNIVERSITY

OREGON STATE UNIVERSITY

UNIVERSITY OF OREGON

OSAKA UNIVERSITY

\author{
UNIVERSITY OF SOUTHERN CALIFORNIA \\ STANFORD UNIVERSITY \\ UNIVERSITY OF TOKYO \\ UNIVERSITY OF UTAH \\ WASHINGTON STATE UNIVERSITY \\ UNIVERSITY OF WASHINGTON

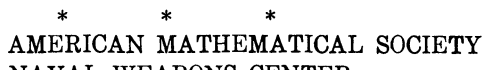 \\ NAVAL WEAPONS CENTER
}

The Supporting Institutions listed above contribute to the cost of publication of this Journal, but they are not owners or publishers and have no responsibility for its content or policies.

Mathematical papers intended for publication in the Pacific Journal of Mathematics should be in typed form or offset-reproduced, (not dittoed), double spaced with large margins. Underline Greek letters in red, German in green, and script in blue. The first paragraph or two must be capable of being used separately as a synopsis of the entire paper. The editorial "we" must not be used in the synopsis, and items of the bibliography should not be cited there unless absolutely necessary, in which case they must be identified by author and Journal, rather than by item number. Manuscripts, in duplicate if possible, may be sent to any one of the four editors. Please classify according to the scheme of Math. Rev. Index to Vol. 39. All other communications to the editors should be addressed to the managing editor, Richard Arens, University of California, Los Angeles, California, 90024.

50 reprints are provided free for each article; additional copies may be obtained at cost in multiples of 50 .

The Pacific Journal of Mathematics is issued monthly as of January 1966. Regular subscription rate: $\$ 48.00$ a year (6 Vols., 12 issues). Special rate: $\$ 24.00$ a year to individual members of supporting institutions.

Subscriptions, orders for back numbers, and changes of address should be sent to Pacific Journal of Mathematics, 103 Highland Boulevard, Berkeley, California, 94708.

PUBLISHED BY PACIFIC JOURNAL OF MATHEMATICS, A NON-PROFIT CORPORATION

Printed at Kokusai Bunken Insatsusha (International Academic Printing Co., Ltd.), 270, 3-chome Totsuka-cho, Shinjuku-ku, Tokyo 160, Japan. 


\section{Pacific Journal of Mathematics}

\section{Vol. 45, No. $2 \quad$ October, 1973}

Kenneth Paul Baclawski and Kenneth Kapp, Induced topologies for quasigroups and loops ............................................. 393

D. G. Bourgin, Fixed point and $\min -\max$ theorems $\ldots \ldots \ldots \ldots \ldots \ldots \ldots$

J. L. Brenner, Zolotarev's theorem on the Legendre symbol ............... 413

Jospeh Atkins Childress, Jr., Restricting isotopies of spheres .............. 415

John Edward Coury, Some results on lacunary Walsh series ................ 419

James B. Derr and N. P. Mukherjee, Generalized Sylow tower groups. II . . . . . . 427

Paul Frazier Duvall, Jr., Peter Fletcher and Robert Allen McCoy, Isotopy Galois

spaces .......................................... 435

Mary Rodriguez Embry, Strictly cyclic operator algebras on a Banach space ... 443

Abi (Abiadbollah) Fattahi, On generalizations of Sylow tower groups ......... 453

Burton I. Fein and Murray M. Schacher, Maximal subfields of tensor products . . 479

Ervin Fried and J. Sichler, Homomorphisms of commutative rings with unit

element .......................................... 485

Kenneth R. Goodearl, Essential products of nonsingular rings ............. 493

George Grätzer, Bjarni Jónsson and H. Lakser, The amalgamation property in

equational classes of modular lattices ...........................

507

$\mathrm{H}$. Groemer, On some mean values associated with a randomly selected simplex

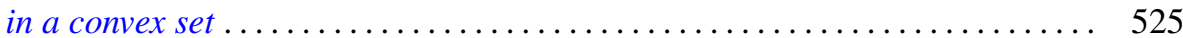

Marcel Herzog, Central 2-Sylow intersections .................... 535

Joel Saul Hillel, On the number of type-k translation-invariant groups ........ 539

Ronald Brian Kirk, A note on the Mackey topology for $\left(C^{b}(X)^{*}, C^{b}(X)\right) \ldots \ldots .543$

J. W. Lea, The peripherality of irreducible elements of lattice.............. 555

John Stewart Locker, Self-adjointness for multi-point differential operators ..... 561

Robert Patrick Martineau, Splitting of group representations ............... 571

Robert Massagli, On a new radical in a topological ring ................. 577

James Murdoch McPherson, Wild arcs in three-space. I. Families of Fox-Artin

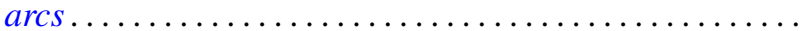

585

James Murdoch McPherson, Wild arcs in three-space. III. An invariant of

oriented local type for exceptional arcs . . . . . . . . . . . . ............ 599

Fred Richman, The constructive theory of countable abelian p-groups ........ 621

Edward Barry Saff and J. L. Walsh, On the convergence of rational functions

which interpolate in the roots of unity ..................

Harold Eugene Schlais, Non-aposyndesis and non-hereditary

decomposability..................................... 643

Mark Lawrence Teply, A class of divisible modules................... 653

Edward Joseph Tully, Jr., H-commutative semigroups in which each

homomorphism is uniquely determined by its kernel ................. 669

Garth William Warner, Jr., Zeta functions on the real general linear group ...... 681

Keith Yale, Cocyles with range $\{ \pm 1\} \ldots \ldots \ldots \ldots \ldots \ldots \ldots \ldots \ldots \ldots \ldots \ldots \ldots . \ldots \ldots$

Chi-Lin Yen, On the rest points of a nonlinear nonexpansive semigroup ........ 699 